\title{
Cuts and penalties: Comment on "Clustering of ultrahigh energy cosmic rays and their sources"
}

\author{
P. G. Tinyakov ${ }^{1,3}$ and I. I. Tkachev ${ }^{2,3}$ \\ ${ }^{1}$ Institute of Theoretical Physics, University of Lausanne, CH-1015 Lausanne, Switzerland \\ ${ }^{2}$ CERN Theory Division, CH-1211 Geneva 23, Switzerland \\ ${ }^{3}$ Institute for Nuclear Research, Moscow 117312, Russia \\ (Received 21 February 2003; published 30 June 2004)
}

\begin{abstract}
In a series of papers we have found statistically significant correlations between arrival directions of ultrahigh energy cosmic rays and BL Lacertae objects. Recently, our calculations were partly repeated by Evans, Ferrer, and Sarkar with different conclusions. We demonstrate that the criticism of Evans, Ferrer, and Sarkar is incorrect. We also present the details of our method.
\end{abstract}

DOI: 10.1103/PhysRevD.69.128301

PACS number(s): 98.70.Sa

\section{INTRODUCTION}

The identification of the sources of the ultrahigh energy cosmic rays (UHECRs) is a key to understanding their nature. The observed small-scale clustering of UHECRs [2-5] suggests that the already existing data $[3,6,7]$ may contain information sufficient to take the first steps in this direction. In a series of papers [8-14] we have shown that there exist significant correlations between the arrival directions of UHECRs and BL Lacertae objects (BL Lacs), and therefore BL Lacs are likely to be sources of UHECRs. Although the present small data set does not allow us to determine with certainty the details of UHECR production and propagation, interesting hints may be obtained [11-14].

The first evidence of a connection between UHECRs and BL Lacs was found in Ref. [8], where we showed that the combined set of Akeino Giant Air Shower Array (AGASA) events with $E>4.8 \times 10^{19} \mathrm{eV}$ and Yakutsk events with $E$ $>2.4 \times 10^{19} \mathrm{eV}$ (identified previously as the sets with largest clustering) correlates strongly with most powerful BL Lacs. The statistical significance of this correlation was found to be $6 \times 10^{-5}$ with a penalty factor included (for explanation of the penalty factor see below).

In the recent paper by Evans, Ferrer, and Sarkar (EFS) [1] the validity of this result was called into question. The rational part of the criticism of EFS boils down to the following two issues: (i) the choice of cuts on BL Lacs and/or calculation of the penalty factor; (ii) the choice of the cosmic ray set. Below we address these issues and demonstrate that the criticism of EFS is unjustified. We also clarify some frequently arising questions related to our analysis.

\section{STATISTICAL SIGNIFICANCE OF CORRELATIONS: CUTS AND PENALTY FACTORS}

\section{A. What is the penalty factor?}

In statistical analysis, one nearly always has to make cuts in order to improve the signal-to-noise ratio. The question is how to take them into account correctly. The common wisdom is that cuts should be fixed a priori, i.e., based on theoretical considerations. In that case the cuts simply limit the number of data points but do not alter the calculation of probabilities.
This is not always possible. For instance, in the case of UHECRs, their acceleration mechanism is not known. How should we impose cuts in a catalogue of astrophysical objects in order to select actual UHECR emitters? In which bandradio, optical, $\mathrm{x}$ ray, $\gamma, \mathrm{TeV}$ ? To what flux limit? In Ref. [8] we proposed an approach to this problem which consists in adjusting cut(s) so as to maximize the signal, and then compensating for this cut adjustment by a penalty factor.

A penalty factor takes into account the "number of independent attempts" made when searching for the best signal. For instance, if two independent catalogues are tried, the penalty factor is 2 (in the limit of small probabilities). If $N$ catalogues tried are not independent, as in the case of cuts in a single catalogue, the penalty factor is smaller than $N$ and should be calculated by means of a Monte Carlo simulation.

In correlation analysis of cosmic rays, the quantity of interest is the probability $p_{\min }^{\text {data }}$ that the observed excess of cosmic rays around source positions is the result of a chance coincidence. This probability depends on cuts made in the source catalogue; the latter are adjusted so that the probability is minimum (correlations are maximum). The cut adjustment should be compensated by a penalty factor which is calculated as follows. A random set of cosmic rays is generated and treated exactly like the real data: the same cuts are tried and the resulting minimum probability $p_{\min }^{\mathrm{MC}}$ is determined. This procedure is then repeated for a large number of random "cosmic ray" sets, and $p_{\min }^{\mathrm{MC}}$ is determined each time. The number of occurrences of a value $p_{\min }^{\mathrm{MC}} \leqslant p$ is then counted as a function of $p$. Divided by the total number of sets, this gives the probability $P(p)$ that the adjustments of cuts produces $p_{\min } \leqslant p$ for a random set of cosmic rays. $P\left(p_{\min }^{\mathrm{data}}\right)$ is the correct measure of the significance of the observed correlations.

When no adjustment of cuts is made one obviously has $P(p)=p$, and the quantitative measure of correlation is the probability $p$ itself. When the cuts are adjusted, small probabilities appear more often by construction, and this relation is modified. The modification can be written in terms of the penalty factor $F(p)$,

$$
P(p)=F(p) \cdot p .
$$

In the presence of cut adjustment, the significance of correlations is determined by the product $F\left(p_{\min }^{\mathrm{data}}\right) \cdot p_{\min }^{\mathrm{data}}$. 
To summarize, all parameters characterizing UHECR sources and UHECRs themselves can be subject to cuts. Some of these cuts can be decided a priori; they reflect (and depend on) the physical assumptions made. These cuts do not require a penalty. One can call these cuts fixed. Alternatively, in the absence of physical arguments, the cut should be chosen (adjusted) so as to maximize the signal. These cuts can be called adjustable. These adjustable cuts imply nontrivial penalty factors; the latter can be calculated in the way explained above.

\section{B. UHECR set}

In our analysis [8], the cosmic ray set was fixed on physical grounds using results of previous publications [4] and was not adjusted in the search for correlations. The motivation was as follows. It was observed earlier $[2,3,5]$ that the highest energy cosmic rays exhibit remarkable property: they autocorrelate on an angular scale consistent with the angular resolution of detectors. The mere existence of these correlations suggests that a relatively small number of point sources of cosmic rays is contributing a considerable fraction of the UHECR flux [15], and that the trace of the original directions to the sources is not lost completely. The identification of these sources via cross-correlation analysis may therefore be possible. Within the hypothesis that UHECR clusters are due to point sources, the strongest correlation signal is expected with the UHECR set which has the most significant autocorrelations. The latter requirement selects [4] AGASA events with $E>4.8 \times 10^{19} \mathrm{eV}$ and Yakutsk events with $E>2.4$ $\times 10^{19} \mathrm{eV}$. The observed angular size of the clusters also fixes the angular scale at which correlations with sources are expected.

The fact that AGASA and Yakutsk sets have different energy cuts in this approach is, of course, disturbing. It may, however, be explained by the different energy calibration of the two experiments or the smaller number of the Yakutsk events. In either case, within our assumptions it is inconsistent to change the energy cuts or to drop the Yakutsk events once autocorrelations are found in this set.

The authors of Ref. [1] have rejected the Yakutsk events because the Yakutsk array has an angular resolution not as good as that of AGASA. By itself, a worse angular resolution does not imply that correlations with sources must be absent in the Yakutsk set: even though the angular resolution is worse, the density of UHECR events around actual sources is larger as compared to a random set, and one has an excess in counts even at small angles. The actual presence of autocorrelations in the Yakutsk data supports this statement. Therefore, it is not correct that "the correlations found at smaller (than Yakutsk angular resolution) angles cannot be meaningful" [1].

\section{Correlations with BL Lacs}

The authors of Ref. [1] have essentially reproduced our Monte Carlo (MC) simulations: the solid curve of Fig. 3 of Ref. [1] agrees with our calculations if the Yakutsk events are discarded. They concluded, however, that we have underestimated the penalty factor. This conclusion was based on (i) a comparison of correlations in the case when one particular set of cuts was chosen to the case when no cuts on BL Lacs were made at all and (ii) smaller correlations of BL Lacs with a different set of UHECRs. Neither such a comparison nor the dependence on the UHECR set has anything to do with the penalty factor. The UHECR set was fixed as described above and was not adjusted during the calculation, thus requiring no penalty. The penalty associated with the adjustment of the BL Lac set should be calculated as described above. This calculation was performed in our paper [8] as follows.

The most complete catalogue of QSO [16] contains 306 confirmed BL Lacs for which the apparent magnitude, redshift (where known), and $6 \mathrm{~cm}$ radio flux are listed (for several objects the $11 \mathrm{~cm}$ radio flux is also given). In Ref. [8] we considered BL Lacs with $z>0.1$ as suggested by the statistics of clustering. (We also examined a complementary small set without further cut adjustment. This "independent attempt" adds 1 to the penalty factor.) We adjusted cuts on the magnitude and $6 \mathrm{~cm}$ radio flux in the following way. First, the grid of $11 \times 11$ cuts was fixed; namely, the cut on magnitude was varied from $m<16$ to $m<20$ with the step 0.4 ; simultaneously the cut on the $6 \mathrm{~cm}$ radio flux was changed from $0.21 \mathrm{Jy}$ to $0.01 \mathrm{Jy}$ with the step $0.02 \mathrm{Jy}$. Note that further "refining" of this set of cuts would not change the results as the sets of BL Lacs obtained would overlap almost completely. The lowest probability (best correlation) found [8] in the real data in this way equals $p_{\mathrm{min}}^{\mathrm{data}}=4 \times 10^{-6} .1$

To calculate the penalty factor associated with cut adjustment, a random configuration of 65 UHECR events was generated. Then its correlation with all $11 \times 11$ subsets of $\mathrm{BL}$ Lacs was examined and the minimum probability $p_{\text {min }}$ over $11 \times 11$ cases was found and recorded. The whole procedure was repeated for the next random configuration. A total of $N_{\text {tot }}=10^{5}$ random configurations were treated in this way. The number of occurrences $N(p)$ of $p_{\min }<p$ was calculated as a function of $p$. Divided by $N_{\text {tot }}$, it determines the corrected probability $P(p)$ and the penalty factor $F(p)$ as defined in Eq. (1). The resulting penalty factor $F(p)$ is shown in Fig. 1. The error bars correspond to statistical errors in determination of $N(p)$.

Taking the penalty factor at the minimum probability obtained for the real data, $F\left(4 \times 10^{-6}\right) \simeq 14$, and adding 1 as a penalty for BL Lacs with $z<0.1$ as explained above, one finds the significance of correlations including penalty as

$$
P\left(4 \times 10^{-6}\right) \simeq 6 \times 10^{-5} .
$$

The significance is $\sim 4 \sigma$ in terms of a Gaussian distribution. It should be pointed out that, in order to get the correct significance, the penalty factor should be multiplied by the minimum probability obtained for the real data with the same set of cuts.

We stress again that the absence of correlations of cosmic rays with the entire sample of known BL Lacs does not mean

\footnotetext{
${ }^{1}$ EFS quote a different number, $2 \times 10^{-5}$. This is merely a misunderstanding of our paper.
} 


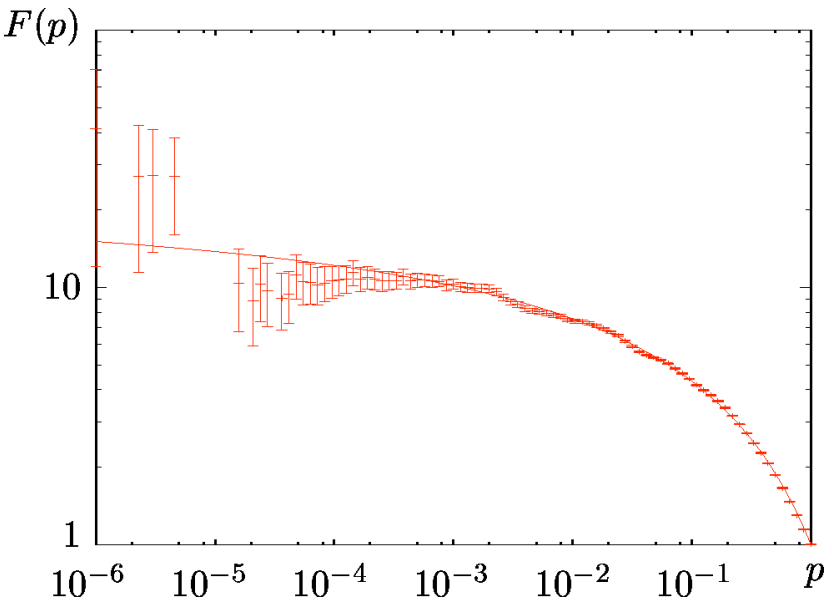

FIG. 1. MC calculation of the penalty factor $P(p)$. Solid line represents the fit by a power law on the log-log scale.

anything. Physically, cosmic rays correlate with the brightest BL Lacs. Including dimmer BL Lacs in the analysis dilutes the correlations, and this is the only conclusion one draws from the analysis made in Ref. [1].

\section{D. "Correlations" with gamma ray bursts}

The authors of Ref. [1] have chosen gamma ray bursts (GRBs) as a control set where correlations are not expected; it has to be compared with the case of BL Lacs. From the fact that correlations with the whole catalogue are absent in both cases while there are many coincidences, the authors of Ref. [1] concluded that GRBs correlate with UHECRs "just as well as do BL Lacs," implying that correlations with BL Lacs are due to cut adjustment. This conclusion can be tested by Monte Carlo simulation: one has to check whether cut adjustment leads to apparent correlations with GRBs. It does not, as we now demonstrate.

The Fourth BATSE Gamma-Ray Burst Catalog [17] contains 1637 objects from which we cut out, in sequential order (according to observation date and time), five nonoverlapping sets of 306 objects each (recall that 306 is the number of confirmed BL Lacs in the catalogue [16]). In each set we have looked for maximum correlations by optimizing cuts. We present two different tests: a one-dimensional cut which allows transparent graphical representation, and a twodimensional cut which mimics more precisely the case of BL Lacs. We took the same maximally autocorrelated UHECR data set as in Refs. [1,8].

In the first test we replace the $11 \times 11$ cuts on the magnitude and radio flux by 121 cuts on the total number of objects (we take them in sequential order, so that first few cuts correspond to including $3,6,9, \ldots$ first objects, and the last cut corresponds to including all 306 objects). ${ }^{2}$ As in the case of BL Lacs, we then calculate the probability of the observed

\footnotetext{
${ }^{2}$ It is not important on which particular parameters the cuts are imposed in the control set. A statement that some parameter is $a$ priori important is already equivalent to the statement that correlations are present.
}

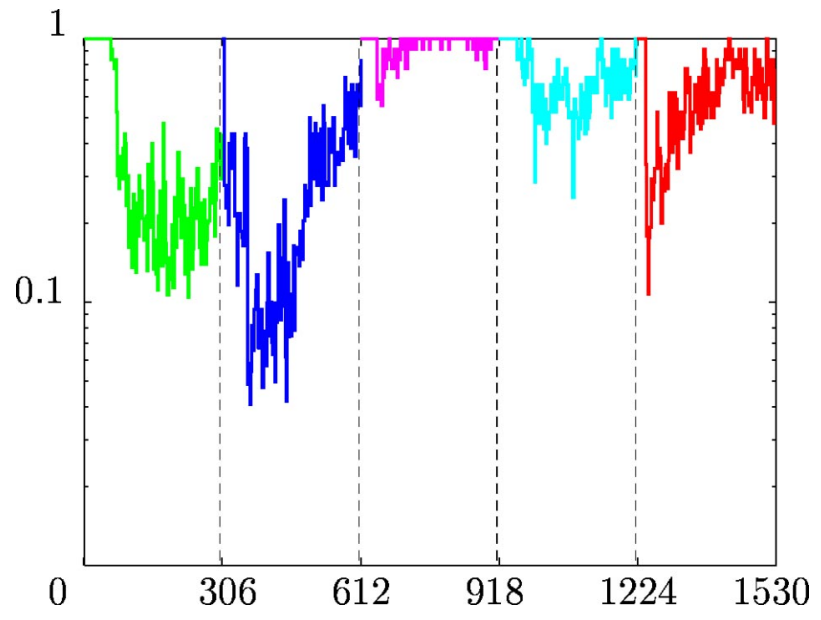

FIG. 2. Correlations of UHECRs with GRBs: probability of the observed excess of UHECR events near GRBs for five independent GRB subsets as a function of the cut applied within each subset.

excess of UHECRs around GRBs as a function of the cut. The results are presented in Fig. 2. The smallest probability found in all five sets is $>4 \%$ (which by itself is not statistically significant). In view of the similar number of cuts in the two cases, Fig. 1 provides an estimate ${ }^{3}$ for the penalty factor, $F(0.04) \approx 5$, in a single set of 306 GRBs. Multiplying the best probability, the penalty factor, and the number of independent GRB sets, one gets a number of order 1 .

To mimic the case of BL Lacs we perform a similar test with two independent cuts. The first cut is done as before on the sequential number of the event. The second cut is imposed on the time (in seconds of the day, UT) of the event. Note that these two cuts are virtually uncorrelated since BATSE registered roughly one event per day. We have searched for the best correlation signal on the grid of 11 $\times 11$ equidistant cuts from 1 to 306 and from 1 to 86400 , respectively. In the above five catalogues consisting of 306 GRBs each, the following lowest probabilities were found: $9.5 \%, 1.4 \%, 20 \%, 1.8 \%, 24 \%$. Multiplying the lowest of these probabilities 0.014 by the penalty factor $F(0.01) \approx 8$ and by the number of independent GRB sets, we again obtain a number of order 1 .

These examples are a good illustration of compensation between the effects due to the cut adjustment and the penalty factor. When compensated by the penalty factor, the cut selection does not introduce apparent correlations when in reality they are absent.

To avoid confusion, we note again that we used GRBs here as a control set, and imposed physically unmotivated cuts on purpose.

\section{SUBTLETIES OF CORRELATION ANALYSIS}

In this section we discuss some subtleties of correlation analysis which were not mentioned in Ref. [1]. We hope that

\footnotetext{
${ }^{3}$ The exact calculation of the penalty factor is, of course, possible along the lines of Sec. II A, but a rough estimate is sufficient in the case at hand.
} 
this will answer a number of frequently arising questions regarding our work and correlation analysis in general.

\section{A. Why completeness of the BL Lac catalogue is not necessary for establishing correlations with UHECRs}

A catalogue of astrophysical objects is called complete when it is believed to contain more than, say, $90 \%$ of all existing objects of a given type, in a given region of the sky, and down to some fixed level of luminosity in a given waveband(s). The catalogue [16], used in our analysis, is not complete simply because it contains all objects known up to date without further selection [18].

It is common wisdom that completeness of the catalogue is crucial for statistical analysis. The reason is obvious: in an incomplete catalogue, the distribution of objects in brightness, position, etc., reflects not only their actual abundance, but also observational bias. When one studies, for instance, the evolution of abundance of some objects with time, one has to make sure that the $\log N / \log S$ dependence reflects actual change in spatial density rather than the fact that remote (and therefore dim) objects are more difficult to observe. Indeed, as it is, the catalogue [16] is not suitable for any analysis where statistical properties of BL Lacs are of interest.

In the correlation analysis of UHECRs the question is different: given a set of candidate sources (BL Lacs in the case at hand), is the distribution of cosmic rays random, or does it peak around BL Lac positions? Clearly, it is the statistical properties of cosmic rays which are of interest in that case, not the statistical properties of BL Lacs. Technically speaking, when calculating the correlation function by the algorithm of Ref. [8], the cosmic ray directions are simulated, while source positions are held fixed. For this reason the method is applicable without change to studying, for instance, a correlation of UHECRs with one particular direction (say, the Galactic center), i.e., in the case when the notion of completeness does not apply.

Completeness is not necessary for establishing the fact of correlations: if statistically significant correlations are found with an incomplete catalogue, this is a real signal. The incompleteness of the catalogue of BL Lacs cannot be a source of correlations with UHECRs. Indeed, the objects which have to be added (removed) from a catalogue to make it complete are absent from (present in) the catalogue for reasons not related to cosmic rays. Therefore, correlations with UHECRs can only be weakened by such an incompleteness.

\section{B. UHECR autocorrelations}

The UHECR set used in our calculations is known to contain event clusters - in fact, it was chosen in such a way that autocorrelations are maximum. When studying cross correlations of such a set with potential sources, one has to be careful to take clusters into account when calculating the probability of chance coincidence; namely, one has to make sure that the signal observed in the data is not due to chance coincidence of clusters of cosmic ray events with candidate sources. For a given cluster, such a coincidence is roughly as probable as for a given single event, but contributes more to the correlation function. This could produce artificial enhancement of correlations if not taken into account in the Monte Carlo simulation.

In our calculations [8] this problem is solved by introducing in each Monte Carlo cosmic ray set the same number of doublets and triplets as there are in the real data. Then chance coincidences between clusters and candidate sources happen in simulated sets as often as in the real data, and thus are correctly accounted for. This is confirmed by calculations presented in Sec. II D, where correlations with GRB catalogues do not appear despite autocorrelations in the UHECR data set.

\section{Choice of angular scale}

The observed angular size of clusters suggests the angular scale at which correlations with sources are expected. In our analysis [8] we fixed it to the previously published $[2,3]$ AGASA value of $2.5^{\circ}$. This value is treated as not adjustable.

When the angular resolution of the experiment is known, there exists a preferred choice of the angular scale-the scale at which correlations are expected to be maximum. This angle can be determined by means of a Monte Carlo simulation as follows. One has to generate cosmic ray sets which are correlated with sources (taking into account the experimental angular resolution) and then measure the angle at which the correlation signal is maximum. In the case of AGASA events this procedure gives a result very close to $2.5^{\circ}$ (see the dotted curve in Fig. 3 of Ref. [8]), so in fact the choice of Ref. [8] was close to optimal.

Alternatively, one may choose not to fix the angular scale and treat it as a free parameter. Then one should adjust it to maximize the correlation and calculate the corresponding penalty factor. A serious problem of this approach is that the result would depend on the limits within which the angular scale is varied. So finally one would have to input in one or another way the information about the expected angular scale of correlations in order to obtain a definite answer. We do not follow this approach in our calculations.

\section{Hidden penalty}

We believe that we have accounted for all contributions to the penalty factor ("effective number of tries") directly related to our work. Still, the obtained significance may be somewhat overestimated. The point is that we are not the first who are looking for correlations between UHECRs and astrophysical objects. Some of these attempts have been published in the literature; others may never have been reported. All these attempts should contribute, in principle, to the penalty factor. However, it does not seem possible to account correctly for all such contributions.

The way around this problem is obvious. First, only very low values of $P$ should be interpreted as a signal (in our calculations we considered $P<10^{-4}$ to be sufficiently low to report our results). Second, and more important, the results have to be confirmed with a new independent data set. 


\section{FURTHER EVIDENCE}

The correlations found in Ref. [10] for a particular set of UHECRs and BL Lacs suggest, strictly speaking, only that UHECRs and BL Lacs are connected to each other. They say little about the acceleration mechanism, particle nature, and other relevant physical parameters. These questions can be fully addressed only with a much larger data set than is available now. Some attempts can be made, however. The final purpose is to arrive at a consistent picture which incorporates all known features of UHECRs. Here is a sketch of these attempts, each bringing additional evidence of the connection between UHECRs and BL Lacs.

In Ref. [12] it was noted that cuts in the BL Lac catalogue, chosen in Ref. [8] so as to maximize correlations with UHECRs, select automatically $\gamma$-ray loud BL Lacs. When this observation is consistently elaborated, i.e., a subsample of the catalogue [18] is selected on the basis of a single criterion, namely, the cross correlation with the EGRET sources, the resulting subset of $14 \gamma$-ray loud BL Lacs correlates with UHECRs at the level of $10^{-7}$ of chance coincidence. This number cannot, of course, be interpreted as the significance of correlations between UHECRs and BL Lacs because of a posteriori selection; rather, the conclusion is that $\gamma$-ray loudness may be a distinctive feature of those BL Lacs which are UHECR accelerators.

In Ref. [11] an attempt was made to determine the charge composition of UHECRs by reconstructing actual arrival directions of UHECR particles bent in the Galactic magnetic field. The idea was that such a reconstruction should improve correlations of UHECRs with BL Lacs if the latter are the sources. Substantial improvement was indeed observed for particles with the charge +1 , which is an indication of the presence of protons.

In Ref. [13] it was observed that, if correlations of UHECRs and BL Lacs were due to chance coincidence, the coinciding rays would be distributed over the sky randomly, reflecting only the local density of BL Lacs and the exposure of a cosmic ray experiment. Thus, any significant deviation in the distribution of correlating rays over the sky from this expectation speaks in favor of a real physical connection between cosmic rays and BL Lacs. In fact, the UHECRs correlating with BL Lacs form two "spots," with a low probability of occurring by chance [13]. This nonuniformity of the distribution of correlating rays may be due to several factors: (1) anisotropy of the extragalactic magnetic fields at scales of order $500 \mathrm{Mpc}$; (2) poor knowledge of the Galactic magnetic field in some areas of the sky; (3) fluctuations in the space distribution of the nearest sources.

\section{CONCLUSIONS}

According to textbooks, any successful statistical analysis consists in formulation of a "null hypothesis" and its subsequent falsification, at some confidence level, by comparing it to the experimental data. In the case at hand the "null hypothesis" which is being tested is that BL Lacs (and, in par- ticular, any subset of them) and UHECRs are uncorrelated. It takes only one counterexample to disprove a hypothesis, while "pro examples" do not prove its validity. An illustration of this general rule has been discussed in Sec. II: the absence of significant correlations of UHECRs with the whole BL Lac catalogue does not prove that UHECRs and BL Lacs are uncorrelated (i.e., that there is no subset of BL Lacs which are sources of UHECRs and thus correlate with them).

Having found such a counterexample (i.e., a case when correlation is significant), the only thing that can be concluded, to a certain confidence level, is that UHECRs and a particular subset of BL Lacs are correlated. The nature and physical implications of these correlations have to be studied separately by formulating and testing different "null hypotheses." References [11-13] are first attempts in this direction.

\section{ACKNOWLEDGMENTS}

We are grateful to V. Rubakov, D. Semikoz, M. Shaposhnikov, and P. Veron for reading the manuscript and valuable comments. The work of P.T. is supported in part by the Swiss Science Foundation, Grant 20-67958.02.

\section{APPENDIX: STATISTICS OF CLUSTERING AND THE NUMBER OF UHECR SOURCES}

The authors of Ref. [1] have misinterpreted our earlier paper [15] which concerns the statistics of clustering of UHECRs and the number of their sources. In view of the growing confusion we would like to clarify this issue.

In Ref. [1] one reads: "[observed occurrence of clusters of UHECRs] was used to estimate the spatial density of sources to be $6 \times 10^{-3} \mathrm{Mpc}^{-3}$ [15]. This would obviously place stringent constraints on candidate astrophysical sources, e.g., $\gamma$-ray bursts (GRBs) have a spatial density of only $\sim 10^{-5} \mathrm{Mpc}^{-3}$. However, a more careful analysis [19] shows that the uncertainties in this estimate are very large. The true number is $n=2.77_{-2.53(2.70)}^{+96.1(916)} \times 10^{-3} \mathrm{Mpc}^{-3}$ at the $68 \%$ (95\%) C.L.; moreover relaxing the assumptions made, viz., that the sources all have the same luminosity and a spectrum $\propto E^{-2}$, increases the allowed range even further, e.g., to $n=180_{-165(174)}^{+2730(8817)} \times 10^{-3} \mathrm{Mpc}^{-3}$.",

The actual situation is different from what is described in this extract. First, the intrinsic inaccuracy of the estimate was fully acknowledged in Ref. [15]. Its physical reason is obvious: clustering is not sensitive to the number of dim sources. The estimates of Ref. [19] cited above are a good illustration of the point: the upper limits are huge and strongly model dependent.

Second, the main point of Ref. [15] was to show that there exists a model-independent lower bound on the number of sources. This bound is presented in Table I of Ref. [15]: at the $90 \%$ and $99 \%$ confidence levels the number of sources is $n>2.3 \times 10^{-4} \mathrm{Mpc}^{-3}$ and $n>3.2 \times 10^{-5} \mathrm{Mpc}^{-3}$, respectively. This is consistent with the calculations of Ref. [19] performed in particular models. 
[1] W. Evans, F. Ferrer, and S. Sarkar, Phys. Rev. D 67, 103005 (2003).

[2] N. Hayashida et al., Phys. Rev. Lett. 77, 1000 (1996).

[3] M. Takeda et al., Astrophys. J. 522, 225 (1999).

[4] P.G. Tinyakov and I.I. Tkachev, Pisma Zh. Éksp. Teor. Fiz. 74, 3 (2001) [JETP Lett. 74, 1 (2001)].

[5] M. Takeda et al., in Proceedings of 27th International Conference on Cosmic Rays, Copernicus Gesellschaft, Hamburg, Germany, 2001, p. 341.

[6] M. Takeda et al., Phys. Rev. Lett. 81, 1163 (1998); M.A. Lawrence, R.J.O. Reid, and A.A. Watson, J. Phys. G 17, 733 (1991); B. N. Afanasiev et al., in Proceedings of the International Symposium on Extremely High Energy Cosmic Rays: Astrophysics and Future Observatories, edited by M. Nagano (ICRR, Tanashi, Tokyo, Japan, 1996), p. 32.

[7] N. Hayashida et al., Astrophys. J. 522, 225 (1999), Appendix.

[8] P.G. Tinyakov and I.I. Tkachev, Pisma Zh. Éksp. Teor. Fiz. 74, 499 (2001) [JETP Lett. 74, 445 (2001)].

[9] P. Tinyakov and I. Tkachev, "Compact Sources of UHECR," in Proceedings of 27th International Cosmic Ray Conference, Hamburg, Germany, 2001, p. 547, http://www.copernicus.org/ icrc/HE1.05.oral.htm

[10] P.G. Tinyakov and I.I. Tkachev, talk at TAUP 2001: Topics in
Astroparticle and Underground Physics, Assergi, Italy, 2001 [Nucl. Phys. B (Proc. Suppl.) 110, 501 (2002)].

[11] P.G. Tinyakov and I.I. Tkachev, Astropart. Phys. 18, 165 (2002).

[12] D.S. Gorbunov, P.G. Tinyakov, I.I. Tkachev, and S.V. Troitsky, Astrophys. J. Lett. 577, L93 (2002).

[13] P. Tinyakov and I. Tkachev, talk at International Workshop on Extremely High Energy Cosmic Rays, Wako, Japan, 2002, hep-ph/0212223.

[14] P. Tinyakov and I. Tkachev, talk at ICRC 2003, Tsukuba, Japan, 2003, astro-ph/0305363.

[15] S.L. Dubovsky, P.G. Tinyakov, and I.I. Tkachev, Phys. Rev. Lett. 85, 1154 (2000).

[16] M.P. Veron-Cetty and P. Veron, "Quasars and Active Galactic Nuclei," 9th ed., ESO Scientific Report, 2000, http://vizier.ustrasbg.fr/viz-bin/Cat?VII/215

[17] W.S. Paciesas et al., astro-ph/9903205.

[18] This catalogue is regularly updated. The current edition is as follows: M.P. Veron-Cetty and P. Veron, Astron. Astrophys. 374, 92 (2001).

[19] Z. Fodor and S.D. Katz, Phys. Rev. D 63, 023002 (2001). 